\title{
Increased incidence of adult pneumococcal pneumonia during school holiday periods
}

\author{
Priya Daniel ${ }^{1}$, Chamira Rodrigo ${ }^{1}$, Thomas Bewick ${ }^{2}$, Carmen Sheppard ${ }^{3}$, \\ Sonia Greenwood ${ }^{1}$, Tricia M. McKeever ${ }^{4}$, Mary Slack ${ }^{5}$ and Wei Shen Lim ${ }^{1}$
}

Affiliations: ${ }^{1}$ Dept of Respiratory Medicine, Nottingham University Hospitals NHS Trust, Nottingham, UK. ${ }^{2}$ Dept of Respiratory Medicine, Derby Teaching Hospitals NHS Foundation Trust, Derby, UK. ${ }^{3}$ Respiratory and Systemic Infection Laboratory, Public Health England, Microbiology Services Division, London, UK. ${ }^{4}$ Division of Epidemiology, University of Nottingham, Nottingham, UK. ${ }^{5}$ School of Medicine, Griffith University, Queensland, Australia.

Correspondence: Priya Daniel, Dept of Respiratory Medicine, David Evans Building, City Hospital Campus, Nottingham University Hospitals NHS Trust, Hucknall Road, Nottingham, NG5 1PB, UK.

E-mail: priyasosha.danielanuh.nhs.uk

ABSTRACT Child contact is a recognised risk factor for adult pneumococcal disease. Peaks in invasive pneumococcal disease incidence observed during winter holidays may be related to changes in social dynamics. This analysis was conducted to examine adult pneumococcal community-acquired pneumonia (CAP) incidence during school holiday periods.

Between September 2008 and 2013, consecutive adults admitted to hospitals covering the Greater Nottingham area with a diagnosis of CAP were studied. Pneumococcal pneumonia was detected using culture and antigen detection methods.

Of 2221 adults studied, 575 (25.9\%) were admitted during school holidays and $643(29.0 \%)$ had pneumococcal CAP. CAP of pneumococcal aetiology was significantly more likely in adults admitted during school holidays compared to term time (35.3\% versus $26.7 \%$; adjusted OR $1.38,95 \%$ CI $1.11-1.72$, $\mathrm{p}=0.004$ ). Over the 5 -year period, the age-adjusted incidence of hospitalised pneumococcal CAP was higher during school holidays compared to term time (incident rate ratio $1.35,95 \%$ CI $1.14-1.60$, $\mathrm{p}<0.001$ ); there was no difference in rates of all-cause CAP or non-pneumococcal CAP. Reported child contact was higher in individuals with pneumococcal CAP admitted during school holidays compared to term time (42.0\% versus $33.7 \%$, OR 1.43 , 95\% CI $1.00-2.03, \mathrm{p}=0.046)$.

Further study of transmission dynamics in relation to these findings and to identify appropriate intervention strategies is warranted.

@ERSpublications

School holiday periods are associated with an increased incidence of adult pneumococcal community-acquired pneumonia http://ow.ly/JiAb3089Gii

Cite this article as: Daniel P, Rodrigo C, Bewick T, et al. Increased incidence of adult pneumococcal pneumonia during school holiday periods. ERJ Open Res 2017; 3: 00100-2016 [https://doi.org/ 10.1183/23120541.00100-2016].

This article has supplementary material available from openres.ersjournals.com

Received: Sept 212016 | Accepted after revision: Dec 312016

Support statement: This research was derived from an unrestricted investigator-initiated research grant from Wyeth (previously) and Pfizer. Funding information for this article has been deposited with the Crossref Funder Registry.

Conflict of interest: Disclosures can be found alongside this article at openres.ersjournals.com

Copyright $\odot$ ERS 2017. This article is open access and distributed under the terms of the Creative Commons Attribution Non-Commercial Licence 4.0. 


\section{Introduction}

Individual risk factors associated with pneumococcal disease include increasing age, presence of chronic respiratory and/or heart disease, cigarette smoking, immunosuppression and child contact [1-5]. At a population level, winter months in temperate regions are associated with an increased incidence of both invasive pneumococcal disease (IPD) and non-bacteraemic community-acquired pneumonia (CAP) [6-10]. Although poorly understood, this association has been attributed to 1) environmental factors such as humidity, temperature, photoperiod and geographical location, e.g. latitude $[7,8] ; 2$ ) host factors such as vitamin D and melatonin levels [11, 12]; and 3) pathogen factors, such as respiratory viral co-infection $[13,14]$. However, studies assessing temporal changes of pneumococcal disease often use aggregate time periods such as seasons and, as a consequence, may contribute to a type of ecological fallacy in which the effects of other subsets of clinically significant exposures are neglected [12]. Changes in human behaviour and school holiday periods have been demonstrated to have an impact on influenza transmission $[15,16]$. In contrast, despite the recognised association between child contact and adult pneumococcal disease [5], there have been no studies to date assessing the relationship between school holiday periods and hospitalisation with noninvasive pneumococcal CAP in adults.

School holiday periods fundamentally alter child contact patterns. Children not only have less contact with other school-age children, but have more adult close contact as a consequence of alterations in childcare arrangements, vacations and family gatherings [17, 18]; this can be expected to affect disease transmission. We hypothesised that changes in child contact patterns during school holiday periods would result in an increase in adult noninvasive pneumococcal CAP during these periods.

\section{Methods}

\section{Study design}

Between September 2008 and September 2013, we conducted a prospective cohort study of consecutive patients with CAP admitted to two teaching hospitals in Nottingham. Combined, these two hospitals cover the catchment area for acute and emergency admissions in the Greater Nottingham area. Adults $>16$ years were included if they presented with symptoms of a lower respiratory tract infection (at least one of cough, increasing breathlessness, sputum production and fever), had radiographic infiltrates consistent with respiratory infection, and were treated by their clinical team for a diagnosis of CAP. Patients who had been hospitalised in the preceding 10 days or who had a diagnosis of either post-obstructive pneumonia (e.g. from an obstructive bronchogenic tumour) or tuberculosis were excluded. Patient demographics and clinical details were collected by the study team after informed consent was obtained. In the event that patients lacked capacity, patient personal consultees were approached for assent for study inclusion. All study procedures were approved by Nottingham Research Ethics Committee.

\section{Study population}

Urine samples were taken on admission from each individual for microbiological analysis: a BinaxNOW Urine Antigen Detection (UAD) assay (Alere Scarborough, Inc., Scarborough, ME, USA) was performed for pneumococcal C-polysaccharide antigen detection in the local microbiological laboratories whilst the remaining volume of urine was frozen and batch transported to Public Health England's Respiratory and Vaccine Preventable Bacteria Reference Laboratory in Colindale for detection of pneumococcal serotype-specific antigen by a multiplex immunoassay (Bio-Plex). The sensitivity and specificity for pneumococcal detection using the BinaxNOW ${ }^{\circledR}$ method are $74 \%$ and $97 \%$, respectively, and for the Bio-Plex method are $79 \%$ and $99 \%$, respectively $[19,20]$. Isolates of Streptococcus pneumoniae from bacteraemic cases were serotyped by slide agglutination using Statens Serum sera (Statens Serum Institut, Copenhagen, Denmark). Patients were defined as having pneumococcal CAP if 1) pneumococcal urinary antigen was detected, 2) pneumococcal serotype was detected by the Bio-Plex assay, or 3) S. pneumoniae was isolated and serotyped from blood cultures. Data regarding child contact (defined as $\leqslant 16$ years of age) in the 4 weeks prior to admission were obtained from all study patients (see supplementary material). Individuals were considered to have had meaningful child contact if any one of the following applied: household contact with a child, kissing or cuddling a child, cumulative child contact for $\geqslant 8 \mathrm{~h}$ over the prior 4 weeks, and/or those whose occupation involved childcare (e.g. day-care workers, teachers). School holiday and term time dates for the corresponding years were obtained from Nottingham City and County Council records. Population demographics for the Greater Nottingham Area were derived from the UK national census data (2011) [21].

\section{Statistical considerations}

Statistical analyses were performed using Stata/IC 13.1 (StataCorp., College Station, TX, USA). Baseline characteristics and risk factors associated with pneumococcal disease of adults admitted during school holiday periods and term time were compared for categorical variables using Pearson's Chi-squared or Fisher's tests. The following variables were included: age, sex, active malignancy, chronic liver disease, 
congestive cardiac failure, dementia, diabetes, chronic renal disease, alcohol excess, cerebrovascular disease, admission from a care facility, HIV diagnosis, smoking status, chronic obstructive airway disease (COPD), asthma, severity of pneumonia (by CURB65 criteria; one point scored for each of new onset confusion, serum urea $>7 \mathrm{mmol} \cdot \mathrm{L}^{-1}$, respiratory rate $\geqslant 30 \cdot \mathrm{min}^{-1}$, systolic blood pressure $<90 \mathrm{mmHg}$ or diastolic blood pressure $\leqslant 60 \mathrm{mmHg}$, age $\geqslant 65$ years) and child contact status. The independent association between admission during school holidays and diagnosis of CAP of pneumococcal compared to non-pneumococcal aetiology was examined using a multivariable logistic regression model. The included variables in the final model were those significantly associated with a diagnosis of pneumococcal CAP and/or that led to a $>10 \%$ change in the regression co-efficient; likelihood ratio tests were used to accurately fit continuous variables. Seasons were grouped by meteorological definitions: winter (December-February), spring (March-May), summer (June-August) and autumn (September-November). Serotypes were grouped into pneumococcal conjugate vaccine (PCV)-7 types (serotypes $4,6 \mathrm{~B}, 9 \mathrm{~V}, 14,18 \mathrm{C}, 19 \mathrm{~F}, 23 \mathrm{~F}$ ), additional PCV-13 types (serotypes 1, 3, 5, 6A/C, 7F/A, 19A) and "other" non-PCV-13 serotypes. A sensitivity analysis was performed to account for the effect of winter by comparing outcomes with the inclusion and exclusion of winter months, for the effect of child contact by excluding individuals that worked with children and those who had no preceding child contact, and for the effect of age by excluding older individuals (defined as those $\geqslant 65$ years). Daily and weekly incidence rates were calculated and differences in incidence of all-cause, non-pneumococcal and pneumococcal CAP requiring hospital admission in school holiday periods versus term time were compared using a Poisson regression model.

\section{Results}

\section{Study population}

During the study period, 2702 individuals met the eligibility criteria; of these, 284 patients had an alternative final diagnosis and consent was not obtained from a further 194 individuals. In an additional three patients, admission dates fell outside the 5-year study period, leaving 2221 patients for analysis. As is characteristic in the UK, school holiday periods in the Greater Nottingham Area were composed of six main holidays over each academic year. Over the 5 years of the study, a total of 465 days were school holidays. The summer season encompassed 224 holiday days (48.2\%) compared to 119 days (25.6\%) over the winter season.

\section{Baseline characteristics}

The median age of the study population was 71 years (interquartile range (IQR) $56-80$ years); $55.1 \%$ were male. In total, 575 individuals (25.9\%) were admitted during school holiday periods. Pneumococcal CAP was diagnosed in 643 out of 2221 individuals $(29.0 \%)$. The diagnosis of pneumococcal disease was made by blood culture, BinaxNOW ${ }^{\oplus}$ UAD and Bio-Plex positivity in 82 out 643 cases (12.8\%), 399 out of 643 cases $(62.1 \%)$ and 403 out of 643 cases $(62.7 \%)$, respectively. Admissions over the winter season accounted for $274(42.6 \%)$ of pneumococcal CAP cases.

\section{The effect of school holiday periods on pneumococcal community-acquired pneumonia}

Those admitted to hospital during school holiday periods were significantly more likely to have a diagnosis of pneumococcal CAP compared to those admitted during term time (35.3\% versus 26.7\%; OR $1.50,95 \%$ CI $1.22-1.83, \mathrm{p}<0.001$ ). In patients with pneumococcal CAP, there was no difference in baseline demographics or disease severity at admission between those admitted during the school holiday periods compared to those admitted during term time. Whilst fewer patients with obstructive airway diseases were admitted during school holiday periods, no differences in other co-morbid conditions were noted between school holiday periods and term time (table 1). Individuals admitted during school holidays were significantly more likely to report child contact in the preceding month (42.0\% during school holiday periods versus $33.7 \%$ during term time; OR $1.43,95 \%$ CI $1.00-2.03, \mathrm{p}=0.046$ ).

Preceding close contact with children of any age was more strongly associated with a diagnosis of pneumococcal CAP than close contact with pre-school children $<5$ years old (OR 1.84, 95\% CI 1.50-2.26, $\mathrm{p}<0.001$ versus OR $1.52,95 \%$ CI $1.19-1.93, \mathrm{p}=0.001)$. In the multivariable model, the odds of pneumococcal CAP remained higher in those individuals admitted during school holiday periods than in those admitted during term time (adjusted OR 1.38, 95\% CI 1.11-1.72, p=0.004). Other factors independently associated with an increased risk of pneumococcal CAP were younger age, smoking status of the patient, alcohol excess, COPD, increasing disease severity at admission and preceding child contact (table 2). In total, 32 individuals worked with children; in a sensitivity analysis excluding these individuals, an increased effect size in the odds of pneumococcal CAP in patients admitted during the school holiday period was observed (adjusted OR 1.70, 95\% CI 1.25-2.31, p=0.001). Conversely, inclusion of only those individuals without preceding child contact reduced the effect of school holidays on pneumococcal disease (adjusted OR 1.29, 95\% CI $0.98-1.69, \mathrm{p}=0.069$ ). In a separate sensitivity analysis, exclusion of patients 
TABLE 1 Baseline characteristics of adults admitted with pneumococcal community-acquired pneumonia during school holiday periods versus term time

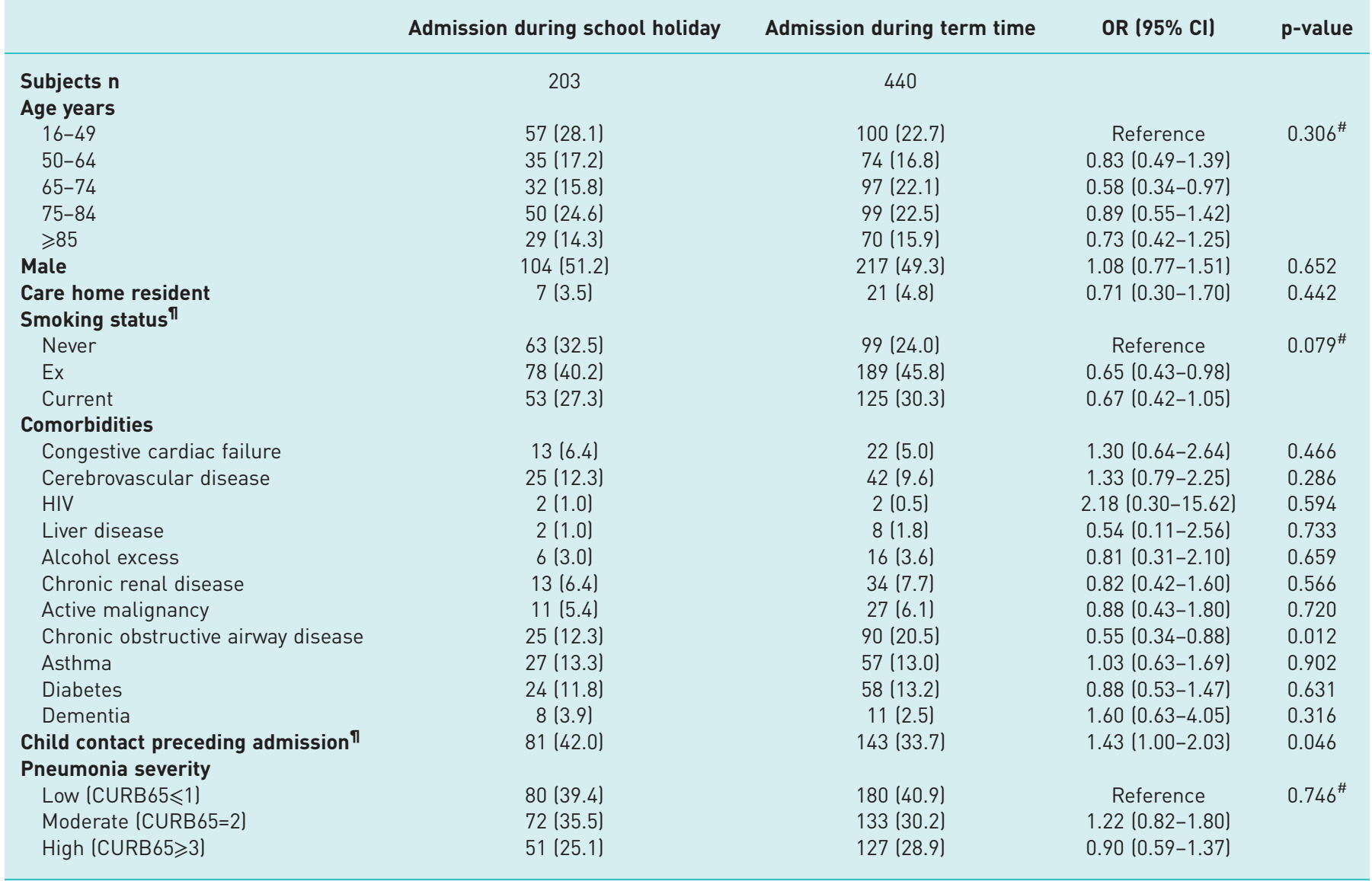

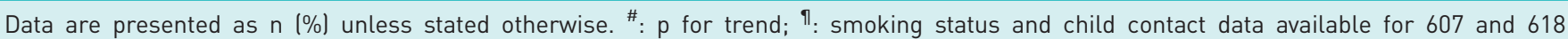
individuals respectively.

\begin{tabular}{|c|c|c|c|}
\hline & AOR & $95 \% \mathrm{Cl}$ & p-value \\
\hline Admission during school holidays & 1.38 & $1.11-1.72$ & 0.004 \\
\hline \multicolumn{4}{|l|}{ Age years } \\
\hline $16-49$ & 1.00 & Reference & \multirow[t]{5}{*}{$0.046^{\#}$} \\
\hline $50-64$ & 0.61 & $0.44-0.84$ & \\
\hline $65-74$ & 0.70 & $0.50-0.97$ & \\
\hline $75-84$ & 0.60 & $0.43-0.84$ & \\
\hline$\geqslant 85$ & 0.68 & $0.46-0.99$ & \\
\hline Male & 0.70 & $0.57-0.86$ & 0.001 \\
\hline \multicolumn{4}{|l|}{ Smoking history } \\
\hline Never & 1.00 & Reference & \multirow[t]{3}{*}{$0.002^{\#}$} \\
\hline Ex & 1.21 & $0.93-1.58$ & \\
\hline Current & 1.57 & $1.18-2.08$ & \\
\hline Alcohol excess & 2.47 & $1.26-4.85$ & 0.009 \\
\hline Chronic obstructive airway disease & 0.75 & $0.58-0.98$ & 0.037 \\
\hline Severity at admission (per point increase on CURB score, excluding age) & 1.63 & $1.44-1.84$ & $<0.001$ \\
\hline Child contact preceding admission & 1.83 & $1.46-2.30$ & $<0.001$ \\
\hline
\end{tabular}


$\geqslant 65$ years old led to an increase in the effect of school holiday periods on pneumococcal disease (adjusted OR 1.52, 95\% CI 1.07-2.17, $\mathrm{p}=0.021$ ).

Of the 643 individuals with pneumococcal CAP, serotypes were obtained in 429 cases. Additional PCV-13 serotypes were the most common serotypes identified in those admitted both in school holiday periods $(\mathrm{n}=68,50.7 \%)$ and term time $(\mathrm{n}=172,58.3 \%)$. Serotypes not included in PCV-13 made up 24.6\% $(\mathrm{n}=33)$ and $18.3 \%(\mathrm{n}=54)$ of serotyped disease admitted during these respective periods (OR 1.79, 95\% CI $1.15-2.80, \mathrm{p}=0.009$ ) (table 3).

Over the 5-year period of the study, an overall decrease in the weekly incidence of pneumococcal CAP was observed despite seasonal spikes over the winter periods (figure 1). After adjustment for age, school holiday periods were associated with an increased incidence of pneumococcal CAP requiring hospital admission (incident rate ratio (IRR) $1.35,95 \%$ CI 1.14-1.60, p<0.001) compared to term time.

There was no difference in rates of all-cause CAP or non-pneumococcal CAP during school holidays periods (IRR 1.02, 95\% CI 0.93-1.13, p=0.636 and IRR 0.90, 95\% CI 0.80-1.01, p=0.087, respectively). In a sensitivity analysis, a non-significant increase in the incidence of pneumococcal CAP during the school holiday periods remained when data from the winter months were excluded (IRR 1.14, 95\% CI 0.91-1.42, p=0.267).

\section{Discussion}

The main finding of this study was that individuals admitted to hospital with a diagnosis of CAP during school holiday periods were significantly more likely to report child contact prior to hospital admission and significantly more likely to have pneumococcal infection compared to adults admitted during term time. A corresponding increase in the incidence of pneumococcal CAP during school holidays was observed.

Children are the predominant nasopharyngeal carriers of $S$. pneumoniae and exposure of adults to children is a recognised risk factor for acquisition of adult pneumococcal disease [2, 5, 22, 23]. Patterns of child contact vary according to changes in human behaviour; at a population level, school holidays are major modifiers of such social patterns [18]. The effect of this modification of social contact patterns is reflected by corresponding changes in transmission rates of viral pathogens via the respiratory route, e.g. influenza, measles and chickenpox, in school holidays compared to term time [24-26].

To our knowledge, this is the first description of the impact of school holidays on the incidence of adult pneumococcal pneumonia. In an epidemiological analysis of episodes of IPD from the US Active Bacterial Core surveillance cohort, WALTER et al. reported annual spikes in the incidence of IPD occurring during winter holiday periods in adults $>50$ years old; the effect was most marked for the pre-PCV-7 vaccine period [27]. An important limitation of that analysis was the lack of individual patient data regarding child contact and the limitation to invasive disease. Other studies investigating temporal changes in pneumococcal disease have aggregated data by season and have generally confirmed the well-recognised association of increased pneumococcal infections during the winter season. However, none of these studies have isolated the effects of school holiday periods on pneumococcal disease. Our results demonstrate that whilst the effect size of school holidays on the incidence of adult pneumococcal disease was highest over the winter season, the effect was observed throughout the year over all 5 years of the study. The absence of a significant relationship between non-pneumococcal CAP and school holiday periods make it unlikely that changes in healthcare-seeking behaviour are responsible for these results.

Previous studies have found that in adults with household child contact, both IPD and nasopharyngeal carriage of S. pneumoniae are more likely to occur, whilst increased duration of contact with children in day care increases the odds of adult carriage $[2,28,29]$. We observed a significantly higher proportion of

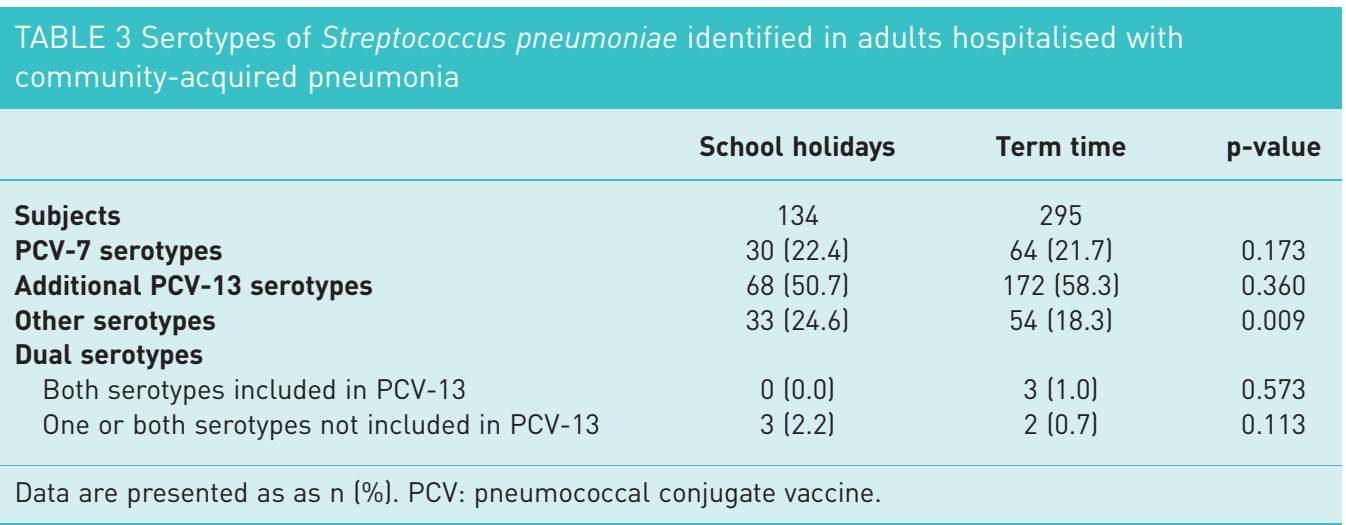



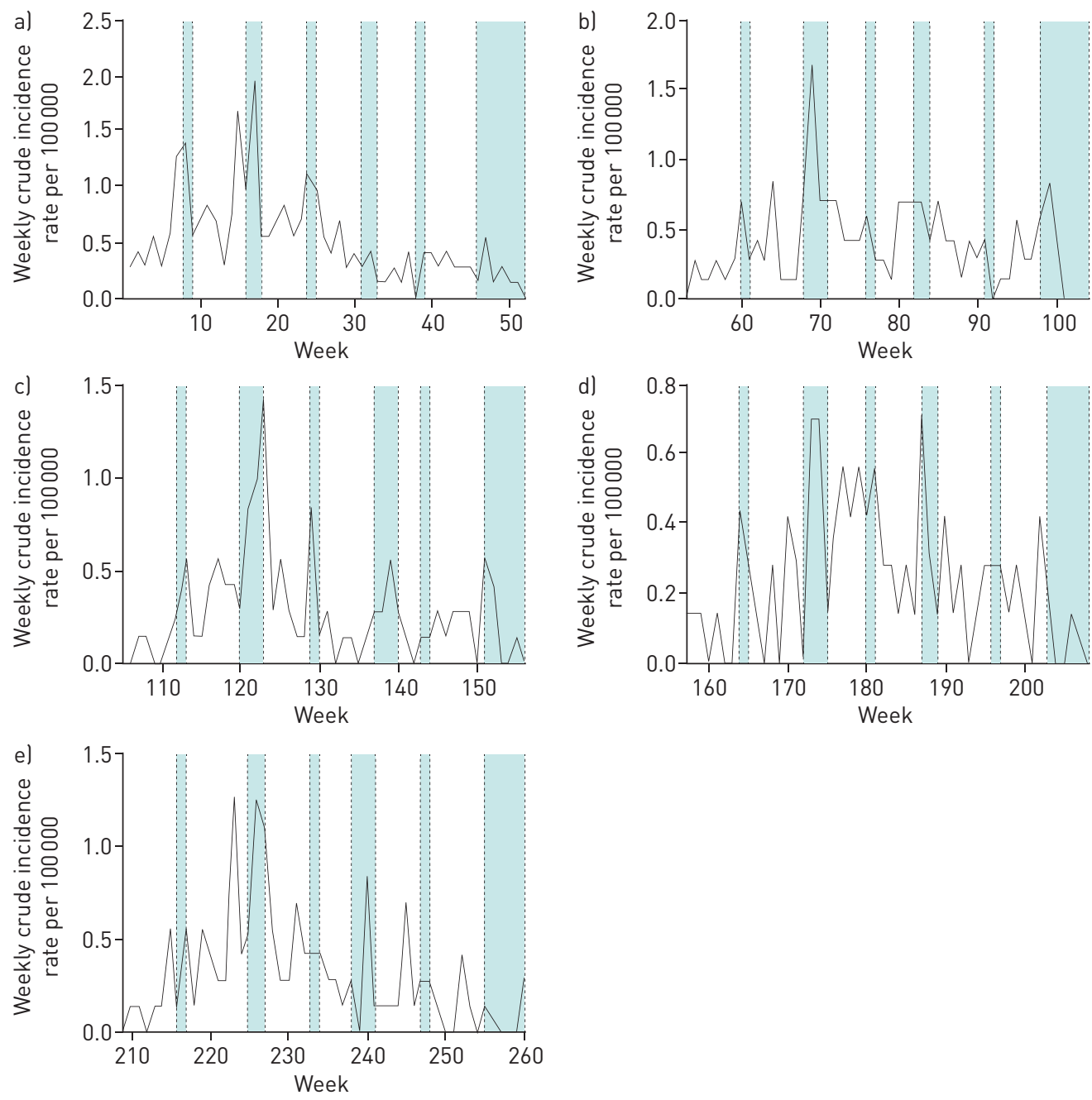

FIGURE 1 Weekly pneumococcal community-acquired pneumonia incidence rates over 5 years, with delineated school holiday periods by year. al Year 1 (September 2008-2009). b) Year 2 (September 2009-2010). c) Year 3 (September 2010-2011). d) Year 4 (September 2011-2012). e) Year 5 (September 2012-2013). The blue shaded areas represent school holiday periods; unshaded areas represent term time.

adult pneumonias due to non-PCV-13 serotypes during school holiday periods compared to term time. This may reflect a serotype shift in pneumococcal carriage in children with transmission to adults during school holidays as a result of increased child-adult contact [30-32].

The effect size of preceding child contact on adult pneumococcal disease was larger when preceding child contact was not restricted to young children $(<5$ years old $)$ only. This is consistent with observations demonstrating that carriage rates, whilst highest in the second to fifth years of life, can persist in $9-22 \%$ of those $>5$ years old $[22,23,33,34]$.

\section{Strengths and limitations of this analysis}

A consistent obstacle in investigating the effect of school holidays is the variable timing of holidays, differing by year and region [35]. We were able to account for staggered school holiday periods over several years to accurately assess the effect on pneumococcal disease incidence in this region. Because the study hospitals covered the catchment area for emergency and acute admissions in the Greater Nottingham area, incidence rates could be calculated whilst minimising selection bias. In addition, the use of multiple laboratory techniques allowed for improved detection of noninvasive pneumococcal disease, which accounts for the vast majority of adult pneumococcal infections. Limitations of this analysis include the lack of contemporaneous data on the pneumococcal carriage state of child close contacts and on pneumococcal pneumonia occurring outside the hospital setting. Although individual patient-level data on the occurrence of child contact was a strength of this study, the duration and intensity of child contact was not measured in each instance. Unaccounted differences in the nature of child contact during school 
holiday periods compared to term time may therefore explain our finding of an independent association of admission during school holidays with adult pneumococcal infection despite adjustment for reported child contact. There is some suggestion that the elderly may act as a reservoir for pneumococcal infection, though strong data to support this hypothesis have not yet emerged. We were unable to explore this in our analysis because contact data with the elderly were not obtained. In addition, although we did not have data on the timing of preceding child contact, the short incubation period (1-3 days) [36] of $S$. pneumoniae makes it unlikely that $S$. pneumoniae acquisition occurred prior to school holidays, which had a minimum duration of 7 days. We recognise that misclassification bias may occur at the very start and end of school holiday periods; however, the size of any such misclassification was found to be very small and therefore unlikely to affect study findings (data not shown). Finally, although we examined seasonal effects on pneumococcal CAP, we did not adjust for other temporal factors, such as environmental temperature, humidity and photoperiod.

Rates of hospital admission for the management of CAP admissions are increasing over time: in the USA alone, a 96\% increase in hospitalisation for pneumococcal pneumonia has been predicted by 2040 [37]. We have observed peaks in adult pneumococcal pneumonia during school holidays despite high infant pneumococcal vaccination coverage (by 2013, 93-96\% of children in the Nottingham City and County Primary Care Trusts had received PCV vaccination by the age of 12 months) [38]. Further studies are required to elucidate the exact reasons for these pneumococcal peaks and, if attributable to increased transmission (child-adult or adult-adult), to identify appropriate interventions to limit such transmission.

\section{Acknowledgements}

The authors would like to thank Tim Harrison from RVPBRU, Colindale, for his support; Sally-Ann Nguyen, Christine More and Seyi Eletu from RVPBRU, Colindale, and Robert Cave, Andrew Shelton, Adrian Patrick, Michelle Stannard and Joanne Palfreyman from the Microbiology Department, Nottingham University Hospitals, for processing the urine samples; clinicians and staff of Nottingham University Hospitals NHS Trust; and Gemma Thompson, Emily Jarvis, Melanie Caine and Gaynor Bates for assisting with patient recruitment. The authors acknowledge the support of Alere in providing BinaxNOW test kits towards the conduct of this study.

\section{References}

1 Torres A, Blasi F, Dartois N, et al. Which individuals are at increased risk of pneumococcal disease and why? Impact of COPD, asthma, smoking, diabetes, and/or chronic heart disease on community-acquired pneumonia and invasive pneumococcal disease. Thorax 2015; 70: 984-989.

2 Nuorti JP, Butler JC, Farley MM, et al. Cigarette smoking and invasive pneumococcal disease. Active Bacterial Core Surveillance Team. N Engl J Med 2000; 342: 681-689.

3 Vila-Corcoles A, Aguirre-Chavarria C, Ochoa-Gondar O, et al. Influence of chronic illnesses and underlying risk conditions on the incidence of pneumococcal pneumonia in older adults. Infection 2015; 43: 699-706.

4 Lipsky BA, Boyko EJ, Inui TS, et al. Risk factors for acquiring pneumococcal infections. Arch Intern Med 1986; 146: 2179-2185.

5 Rodrigo C, Bewick T, Sheppard C, et al. Pneumococcal serotypes in adult non-invasive and invasive pneumonia in relation to child contact and child vaccination status. Thorax 2014; 69: 168-173.

6 Ampofo K, Bender J, Sheng X, et al. Seasonal invasive pneumococcal disease in children: role of preceding respiratory viral infection. Pediatrics 2008; 122: 229-237.

7 Dowell SF, Whitney CG, Wright C, et al. Seasonal patterns of invasive pneumococcal disease. Emerg Infect Dis J 2003; 9: 574-579.

8 Kim PE, Musher DM, Glezen WP, et al. Association of invasive pneumococcal disease with season, atmospheric conditions, air pollution, and the isolation of respiratory viruses. Clin Infect Dis 1996; 22: 100-106.

9 Herrera-Lara S, Fernández-Fabrellas E, Cervera-Juan A, et al. Do seasonal changes and climate influence the etiology of community acquired pneumonia? Arch Bronconeumol 2013; 49: 140-145.

10 Lieberman D, Lieberman D, Friger MD. Seasonal variation in hospital admissions for community-acquired pneumonia: a 5-year study. J Infect 1999; 39: 134-140.

11 Dowell SF. Seasonal variation in host susceptibility and cycles of certain infectious diseases. Emerg Infect Dis 2001; 7: 369-374.

12 Fisman DN. Seasonality of infectious diseases. Annu Rev Public Health 2007; 28: 127-143.

13 Kuster SP, Tuite AR, Kwong JC, et al. Evaluation of coseasonality of influenza and invasive pneumococcal disease: results from prospective surveillance. PLoS Med 2011; 8: e1001042.

14 Talbot TR, Poehling KA, Hartert TV, et al. Seasonality of invasive pneumococcal disease: temporal relation to documented influenza and respiratory syncytial viral circulation. Am J Med 2005; 118: 285-291.

15 Cauchemez S, Ferguson NM, Wachtel C, et al. Closure of schools during an influenza pandemic. Lancet Infect Dis 2009; 9: 473-481.

16 Earn DJ, He D, Loeb MB, et al. Effects of school closure on incidence of pandemic influenza in Alberta, Canada. Ann Intern Med 2012; 156: 173-181.

17 Rohani P, Earn DJ, Finkenstädt B, et al. Population dynamic interference among childhood diseases. Proc Biol Sci 1998; 265: 2033-2041.

18 Eames KT, Tilston NL, Edmunds WJ. The impact of school holidays on the social mixing patterns of schoo children. Epidemics 2011; 3: 103-108.

19 Sinclair A, Xie X, Teltscher M, et al. Systematic review and meta-analysis of a urine-based pneumococcal antigen test for diagnosis of community-acquired pneumonia caused by Streptococcus pneumoniae. J Clin Microbiol 2013; 51: 2303-2310. 

beads for detection of serotype-specific streptococcus pneumoniae antigen in urine samples. J Med Microbiol 2011; 60: 49-55.

21 Office for National Statistics. 2011 Census. www.ons.gov.uk/ons/guide-method/census/2011/index.html?utm source=twitterfeed\&utm_medium=twitter Date last accessed: March 1, 2016.

22 Regev-Yochay G, Raz M, Dagan R, et al. Nasopharyngeal carriage of Streptococcus pneumoniae by adults and children in community and family settings. Clin Infect Dis 2004; 38: 632-639.

23 Bogaert D, van Belkum A, Sluijter M, et al. Colonisation by Streptococcus pneumoniae and Staphylococcus aureus in healthy children. Lancet 2004; 363: 1871-1872.

24 Jackson C, Mangtani P, Fine P, et al. The effects of school holidays on transmission of varicella zoster virus, England and Wales, 1967-2008. PLoS One 2014; 9: e99762.

25 Fine PE, Clarkson JA. Measles in England and Wales--I: an analysis of factors underlying seasonal patterns. Int $J$ Epidemiol 1982; 11: 5-14.

26 Cauchemez S, Valleron AJ, Boelle PY, et al. Estimating the impact of school closure on influenza transmission from Sentinel data. Nature 2008; 452: 750-754.

27 Walter ND, Taylor TH, Dowell SF, et al. Holiday spikes in pneumococcal disease among older adults. $N$ Engl $J$ Med 2009; 361: 2584-2585.

28 Huang SS, Finkelstein JA, Lipsitch M. Modeling community- and individual-level effects of child-care center attendance on pneumococcal carriage. Clin Infect Dis 2005; 40: 1215-1222.

29 Hendley JO, Sande MA, Stewart PM, et al. Spread of Streptococcus pneumoniae in families. I. Carriage rates and distribution of types. J Infect Dis 1975; 132: 55-61.

30 Moore MR, Link-Gelles R, Schaffner W, et al. Effect of use of 13-valent pneumococcal conjugate vaccine in children on invasive pneumococcal disease in children and adults in the USA: analysis of multisite, population-based surveillance. Lancet Infect Dis 2015; 15: 301-309.

31 Harboe ZB, Dalby T, Weinberger DM, et al. Impact of 13-valent pneumococcal conjugate vaccination in invasive pneumococcal disease incidence and mortality. Clin Infect Dis 2014; 59: 1066-1073.

32 Rodrigo C, Bewick T, Sheppard C, et al. Impact of infant 13-valent pneumococcal conjugate vaccine on serotypes in adult pneumonia. Eur Respir J 2015; 45: 1632-1641.

33 Marchisio P, Esposito S, Schito GC, et al. Nasopharyngeal carriage of Streptococcus pneumoniae in healthy children: implications for the use of heptavalent pnemococcal conjugate vaccine. Emerg Infect Dis 2002; 8: 479-484.

34 van Hoek AJ, Sheppard CL, Andrews NJ, et al. Pneumococcal carriage in children and adults two years after introduction of the thirteen valent pneumococcal conjugate vaccine in England. Vaccine 2014; 32: 4349-4355.

35 te Beest DE, van Boven M, Hooiveld M, et al. Driving factors of influenza transmission in the Netherlands. Am J Epidemiol 2013; 178: 1469-1477.

36 Public Health England. Pneumococcal: the green book, chapter 25. In: Immunisation against Infectious Disease. London, Public Health England, 2013; pp. 295-314. www.gov.uk/government/publications/pneumococcal-thegreen-book-chapter-25 Date last accessed: March 1, 2016. Date last updated: December 4, 2013.

37 Wroe PC, Finkelstein JA, Ray GT, et al. Aging population and future burden of pneumococcal pneumonia in the United States. J Infect Dis 2012; 205: 1589-1592.

38 Public Health England. Quarterly Vaccine Coverage Data Tables. http://webarchive.nationalarchives.gov.uk/ 20140629102627/http://www.hpa.org.uk/webw/HPAweb\&HPAwebStandard/HPAweb_C/1211441442288 Date last accessed: March 1, 2016. 